\title{
Solving a modified nonlinear epidemiological model of computer viruses by homotopy analysis method
}

\author{
Samad Noeiaghdam ${ }^{1}$ (1) $\cdot$ Muhammad Suleman $^{2,3} \cdot$ Hüseyin Budak $^{4}$
}

Received: 9 April 2018 / Accepted: 29 August 2018/ Published online: 7 September 2018

(C) The Author(s) 2018

\begin{abstract}
The susceptible-infected-recovered model of computer viruses is investigated as a nonlinear system of ordinary differential equations by using the homotopy analysis method (HAM). The HAM is a flexible method which contains the auxiliary parameters and functions. This method has an important tool to adjust and control the convergence region of obtained solution. The numerical solutions are presented for various iterations, and the residual error functions are applied to show the accuracy of presented method. Several $\hbar$-curves are plotted to demonstrate the regions of convergence, and the residual errors are obtained for different values of theses regions.
\end{abstract}

Keywords Susceptible-infected-recovered model $\cdot$ Modified epidemiological model $\cdot$ Computer virus $\cdot$ Homotopy analysis method $\cdot \hbar$-Curve

\section{Introduction}

In recent decades, using the computer systems has led to great changes in many fields of life. These changes are many important and wonderful where we cannot leave applying the computer systems.

Computer viruses are a malicious software program that can be written with different aims. These softwares help the user to enter the victim computer without permission. The virus should never be considered to be harmless and remain in the system. There are several types of viruses that can be categorized according to their source, technique, file type that infects, where they are hiding, the type of damage they enter, the type of operating system or the design on which they are attacking. Several computer viruses such as

Samad Noeiaghdam

s.noeiaghdam.sci@iauctb.ac.ir;

samadnoeiaghdam@gmail.com

1 Department of Mathematics, Central Tehran Branch, Islamic Azad University, Tehran, Iran

2 Faculty of Science, Jiangsu University, Zhenjiang, China

3 Department of Mathematics, Comsats Institute of Information Technology, Islamabad, Pakistan

4 Department of Mathematics, Faculty of Science and Arts, Düzce University, Düzce, Turkey
ILOVEYOU, Melissa, My Doom, Code Red, Sasser have been recognized. Also, in recent years, the Stuxnet virus targeted the Siemens hardware and software industry. Claims and statements allege that the virus was part of spy campaign to hit Iran's nuclear plant, Natanz. These viruses have been able to infect thousands of computers and have hurt billions dollar in computers around the world.

Thus, scientists start to work on finding methods to analyze, track, model and protect against viruses. Computer viruses are similar to biological viruses, and we can study it in two case, microscopic and macroscopic models. Therefore, many studies have proposed solutions that help us to understand how computer viruses operate. In order to control the growth and reproduction of computer viruses, many dynamical model have been presented $[7,18,19,29,33-35,40,42]$. In this study, the following modified SIR model of computer viruses

$$
\begin{aligned}
& \frac{\mathrm{d} S(t)}{\mathrm{d} t}=f_{1}-\lambda S(t) I(t)-\mathrm{d} S(t), \\
& \frac{\mathrm{d} I(t)}{\mathrm{d} t}=f_{2}+\lambda S(t) I(t)-\varepsilon I(t)-\mathrm{d} R(t), \\
& \frac{\mathrm{d} R(t)}{\mathrm{d} t}=f_{3}+\varepsilon I(t)-\mathrm{d} R(t) .
\end{aligned}
$$

is illustrated where the initial conditions are in the following form 


$$
\begin{aligned}
& S(0)=S_{0}(t)=S_{0}, \\
& I(0)=I_{0}(t)=I_{0}, \\
& R(0)=R_{0}(t)=R_{0} .
\end{aligned}
$$

In recent years, many applicable methods have been applied to solve the linear and nonlinear problems [13-16, 27, 28, 31, 39, 41]. Also, many numerical and semi-analytical methods presented to solve the nonlinear epidemiological model (1) such as multi-step homotopy analysis method [17], collocation method with Chebyshev polynomials [32] and variational iteration method [29]. In this paper, the HAM [22-26] is applied to solve the nonlinear system of Eq. (1). This method has many applications to solve various problems arising in the mathematics, physics and engineering $[1-6,8-12,14-16$, $20,21,30,31,36-38]$. The $\hbar$-curves are demonstrated to find the region of convergence. It is one of important abilities of HAM that the other methods do not have this ability. In order to show the efficiency and accuracy of presented approach, the residual error functions for different values of $\hbar, m$ are estimated. Several graphs of error functions are demonstrated to show the capabilities of method. List of functions, variables and initial assumptions of system (1) are presented in Table 1.

\section{Main idea}

Let $L_{S}, L_{I}$ and $L_{R}$ are the linear operators which are defined in the following form

$L_{S}=\frac{\mathrm{dS}}{\mathrm{d} t}, L_{I}=\frac{\mathrm{dI}}{\mathrm{d} t}, L_{R}=\frac{\mathrm{dR}}{\mathrm{d} t}$

and for constant values $c_{1}, c_{2}$ and $c_{3}$ we obtain

$$
L_{S}\left(c_{1}\right)=0, L_{I}\left(c_{2}\right)=0, L_{R}\left(c_{3}\right)=0 .
$$

Now, the following homotopy maps can be defined as
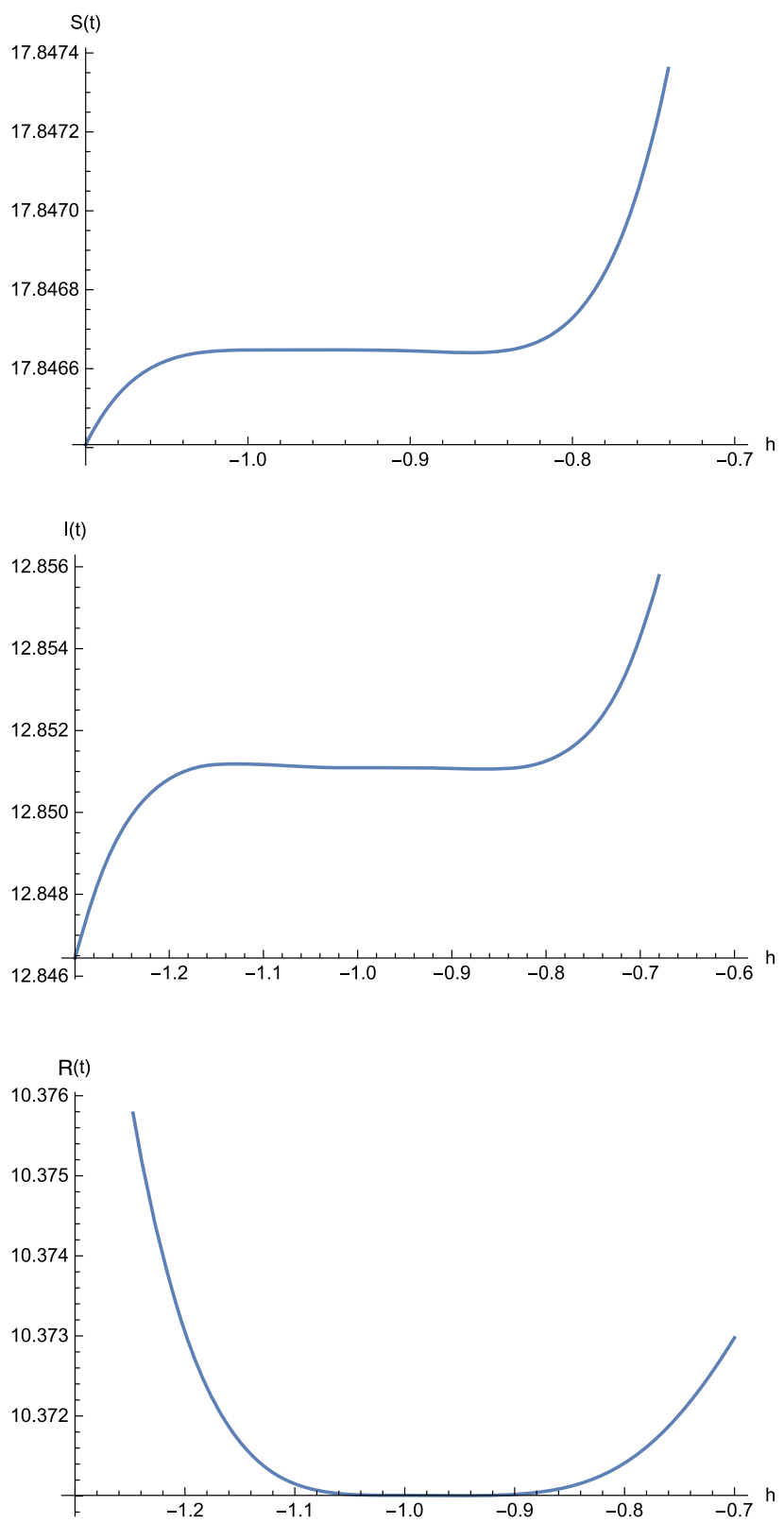

Fig. $1 \hbar$-curves of $S(t), I(t)$ and $R(t)$ for $m=5, t=1$
Table 1 List of parameters and functions

\begin{tabular}{lll}
\hline Parameters and functions & Meaning & Values \\
\hline$S(t)$ & Susceptible computers at time $t$ & $S(0)=20$ \\
$I(t)$ & Infected computers at time $t$ & $I(0)=15$ \\
$R(t)$ & Recovered computers at time $t$ & $R(0)=10$ \\
$f_{1}, f_{2}, f_{3}$ & Rate of external computers connected to the network & $f_{1}=f_{2}=f_{3}=0$ \\
$\lambda$ & Rate of infecting for susceptible computer & $\lambda=0.001$ \\
$\varepsilon$ & Rate of recovery for infected computers & $\varepsilon=0.1$ \\
$d$ & Rate of removing from the network & $d=0.1$ \\
\hline
\end{tabular}



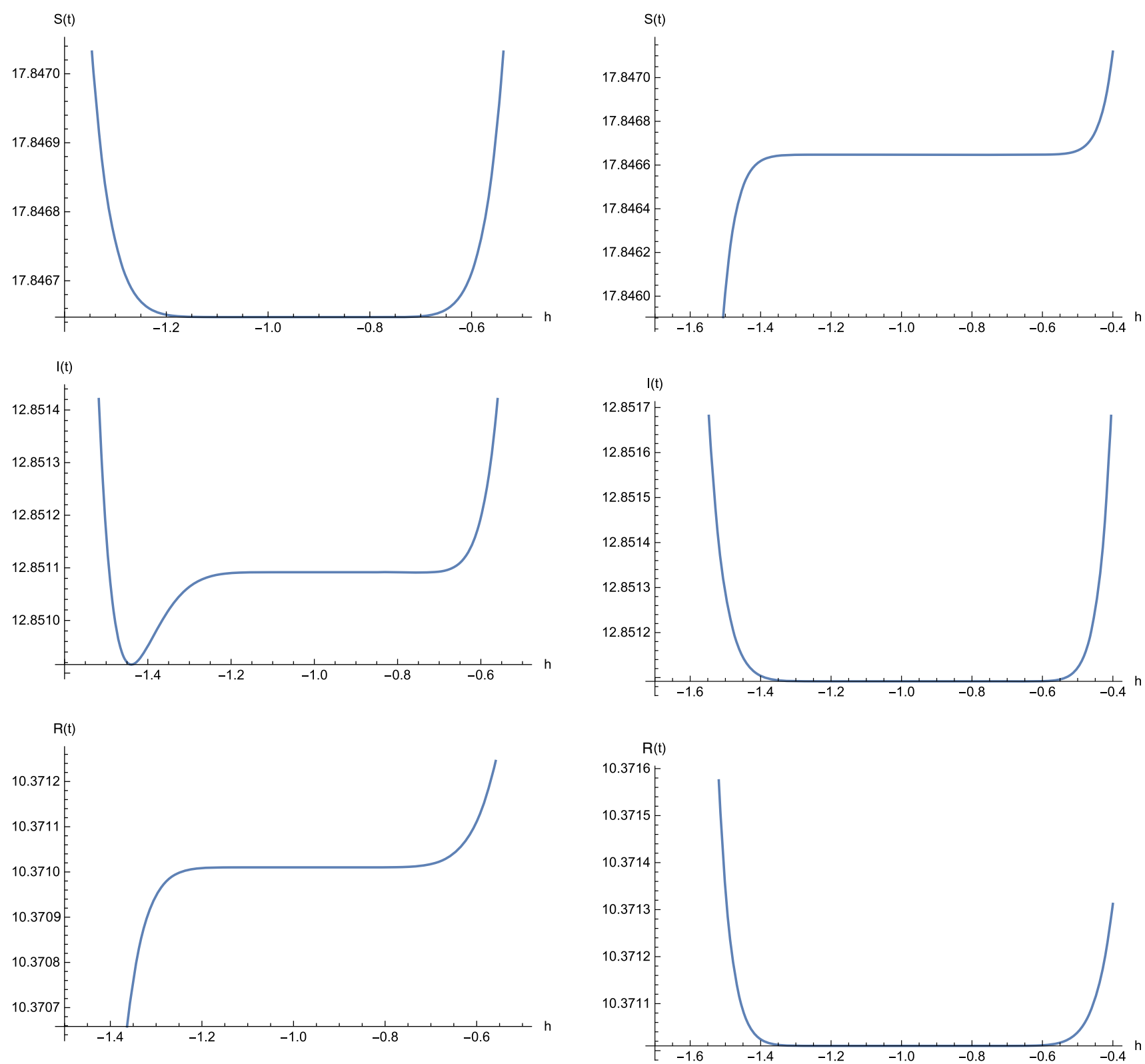

Fig. $2 \hbar$-curves of $S(t), I(t)$ and $R(t)$ for $m=10, t=1$

$$
\begin{aligned}
& H_{S}(\tilde{S}(t ; q), \tilde{I}(t ; q), \tilde{R}(t ; q))=(1-q) L_{S}\left[\tilde{S}(t ; q)-S_{0}(t)\right] \\
& \quad-q \hbar H_{S}(t) N_{S}[\tilde{S}(t ; q), \tilde{I}(t ; q), \tilde{R}(t ; q)], \\
& H_{I}(\tilde{S}(t ; q), \tilde{I}(t ; q), \tilde{R}(t ; q))=(1-q) L_{I}\left[\tilde{I}(t ; q)-I_{0}(t)\right] \\
& \quad-q \hbar H_{I}(t) N_{I}[\tilde{S}(t ; q), \tilde{I}(t ; q), \tilde{R}(t ; q)], \\
& H_{R}(\tilde{S}(t ; q), \tilde{I}(t ; q), \tilde{R}(t ; q))=(1-q) L_{R}\left[\tilde{R}(t ; q)-R_{0}(t)\right] \\
& \quad-q \hbar H_{R}(t) N_{R}[\tilde{S}(t ; q), \tilde{I}(t ; q), \tilde{R}(t ; q)],
\end{aligned}
$$

Fig. $3 \hbar$-curves of $S(t), I(t)$ and $R(t)$ for $m=15, t=1$

operators, and finally $N_{S}, N_{I}$ and $N_{R}$ are the nonlinear operators which are defined as follows

where $q \in[0,1]$ is an embedding parameter, $\hbar \neq 0$ is a convergence control parameter, $H_{S}(t), H_{I}(t)$ and $H_{R}(t)$ are the auxiliary functions, $L_{S}, L_{I}$ and $L_{R}$ are the linear 
Table 2 Regions of convergence and the optimal values of $\hbar$ for $m=5,10,15$ and $t=1$

\begin{tabular}{lllllll}
\hline$m$ & $\hbar_{S}$ & $\hbar_{S}^{*}$ & $\hbar_{I}$ & $\hbar_{I}^{*}$ & $\hbar_{R}$ & $\hbar_{R}^{*}$ \\
\hline 5 & $-1.1 \leq \hbar_{S} \leq-0.8$ & -0.99994 & $-1.2 \leq \hbar_{I} \leq-0.8$ & -1.00204 & $-1.1 \leq \hbar_{R} \leq-0.9$ \\
10 & $-1.2 \leq \hbar_{S} \leq-0.7$ & -1.0036 & $-1.2 \leq \hbar_{I} \leq-0.7$ & -1.0038 & $-1.2 \leq \hbar_{R} \leq-0.99058$ \\
15 & $-1.3 \leq \hbar_{S} \leq-0.5$ & -1 & $-1.3 \leq \hbar_{I} \leq-0.6$ & -1 & $-1.3 \leq \hbar_{R} \leq-0.6$ & -1 \\
\hline
\end{tabular}

$$
\begin{aligned}
& N_{S}[\tilde{S}(t ; q), \tilde{I}(t ; q), \tilde{R}(t ; q)]=\frac{\partial \tilde{S}(t ; q)}{\mathrm{d} t} \\
& \quad-f_{1}+\lambda \tilde{S}(t ; q) \tilde{I}(t ; q)+\mathrm{d} \tilde{S}(t ; q), \\
& N_{I}[\tilde{S}(t ; q), \tilde{I}(t ; q), \tilde{R}(t ; q)]=\frac{\partial \tilde{I}(t ; q)}{\mathrm{d} t}-f_{2} \\
& \quad-\lambda \tilde{S}(t ; q) \tilde{I}(t ; q)+\varepsilon \tilde{I}(t ; q)+\mathrm{d} \tilde{R}(t ; q), \\
& N_{R}[\tilde{S}(t ; q), \tilde{I}(t ; q), \tilde{R}(t ; q)]=\frac{\partial \tilde{R}(t ; q)}{\mathrm{d} t}-f_{3} \\
& \quad-\varepsilon \tilde{I}(t ; q)+\mathrm{d} \tilde{R}(t ; q) .
\end{aligned}
$$

According to [22-24] the zero-order deformation, equations can be defined as

\begin{tabular}{|c|c|c|c|c|c|c|}
\hline$t$ & $\hbar=-1.2$ & $\hbar=-1.1$ & $\hbar=-1$ & $\hbar_{\mathrm{opt}}=-0.99994$ & $\hbar=-0.9$ & $\hbar=-0.8$ \\
\hline 0.0 & 0.000736 & 0.000023 & $2.66454 \times 10^{-15}$ & $2.66454 \times 10^{-15}$ & 0.000023 & 0.000736 \\
\hline 0.2 & 0.00144214 & 0.0000696185 & $1.98321 \times 10^{-11}$ & $3.81375 \times 10^{-11}$ & $2.27788 \times 10^{-6}$ & 0.000388735 \\
\hline 0.4 & 0.00240313 & 0.00014723 & $4.38416 \times 10^{-10}$ & $7.35197 \times 10^{-10}$ & $7.10003 \times 10^{-6}$ & 0.000128218 \\
\hline 0.6 & 0.00366188 & 0.000265063 & $1.87637 \times 10^{-9}$ & $3.38906 \times 10^{-9}$ & $8.84562 \times 10^{-6}$ & 0.0000577341 \\
\hline 0.8 & 0.00526424 & 0.000433423 & $1.93464 \times 10^{-9}$ & $6.74028 \times 10^{-9}$ & $6.1317 \times 10^{-6}$ & 0.000180563 \\
\hline 1.0 & 0.00725902 & 0.000663706 & $1.1876 \times 10^{-8}$ & $9.02851 \times 10^{-11}$ & $1.58842 \times 10^{-6}$ & 0.000250966 \\
\hline$\|E\|$ & 0.00725902 & 0.000663706 & $1.1876 \times 10^{-8}$ & $6.74028 \times 10^{-9}$ & 0.000023 & 0.000736 \\
\hline$t$ & $\hbar=-1.2$ & $\hbar=-1.1$ & $\hbar_{\mathrm{opt}}=-1.00204$ & $\hbar=-1$ & $\hbar=-0.9$ & $\hbar=-0.8$ \\
\hline 0.0 & 0.000704 & 0.000022 & $8.43769 \times 10^{-14}$ & $2.66454 \times 10^{-15}$ & 0.000022 & 0.000704 \\
\hline 0.2 & 0.00079364 & 0.0000221048 & $1.19964 \times 10^{-9}$ & $2.0886 \times 10^{-10}$ & $9.04759 \times 10^{-6}$ & 0.000553202 \\
\hline 0.4 & 0.000675079 & $5.11674 \times 10^{-6}$ & $1.10223 \times 10^{-8}$ & $6.48732 \times 10^{-9}$ & $9.76643 \times 10^{-6}$ & 0.000345977 \\
\hline 0.6 & 0.000291417 & 0.0000717513 & $3.28701 \times 10^{-8}$ & $4.78103 \times 10^{-8}$ & 0.0000295926 & 0.0000980449 \\
\hline 0.8 & 0.000417872 & 0.000191176 & $4.72582 \times 10^{-8}$ & $1.955 \times 10^{-7}$ & 0.000046278 & 0.00017579 \\
\hline 1.0 & 0.00151692 & 0.000378046 & $3.75195 \times 10^{-9}$ & $5.78838 \times 10^{-7}$ & 0.0000563829 & 0.000461654 \\
\hline$\|E\|$ & 0.00151692 & 0.000378046 & $4.72582 \times 10^{-8}$ & $5.78838 \times 10^{-7}$ & 0.0000563829 & 0.000704 \\
\hline$t$ & $\hbar=-1.2$ & $\hbar=-1.1$ & $\hbar=-1$ & $\hbar_{o p t}=-0.99058$ & $\hbar=-0.9$ & $\hbar=-0.8$ \\
\hline 0.0 & 0.00016 & $5 . \times 10^{-6}$ & 0 & $3.70868 \times 10^{-11}$ & $5 . \times 10^{-6}$ & 0.00016 \\
\hline 0.2 & 0.000761715 & 0.0000434312 & $5.69638 \times 10^{-10}$ & $1.31142 \times 10^{-9}$ & 0.000013481 & 0.000148655 \\
\hline 0.4 & 0.00152906 & 0.0000988607 & $1.82284 \times 10^{-8}$ & $1.09745 \times 10^{-8}$ & 0.0000206331 & 0.000383918 \\
\hline 0.6 & 0.00245803 & 0.000169652 & $1.38422 \times 10^{-7}$ & $6.07365 \times 10^{-8}$ & 0.0000172599 & 0.000546984 \\
\hline 0.8 & 0.00354105 & 0.000252826 & $5.83309 \times 10^{-7}$ & $1.10979 \times 10^{-7}$ & $4.58752 \times 10^{-6}$ & 0.000639632 \\
\hline 1.0 & 0.00476692 & 0.000343953 & $1.78012 \times 10^{-6}$ & $1.40369 \times 10^{-9}$ & 0.0000157769 & 0.000664199 \\
\hline$\|E\|$ & 0.00476692 & 0.000343953 & $1.78012 \times 10^{-6}$ & $1.10979 \times 10^{-7}$ & 0.0000206331 & 0.000664199 \\
\hline
\end{tabular}

$$
\begin{aligned}
& (1-q) L_{S}\left[\tilde{S}(t ; q)-S_{0}(t)\right]-q \hbar H_{S}(t) N_{S}[\tilde{S}(t ; q), \tilde{I}(t ; q), \tilde{R}(t ; q)]=0, \\
& (1-q) L_{I}\left[\tilde{I}(t ; q)-I_{0}(t)\right]-q \hbar H_{I}(t) N_{I}[\tilde{S}(t ; q), \tilde{I}(t ; q), \tilde{R}(t ; q)]=0, \\
& (1-q) L_{R}\left[\tilde{R}(t ; q)-R_{0}(t)\right]-q \hbar H_{R}(t) N_{R}[\tilde{S}(t ; q), \tilde{I}(t ; q), \tilde{R}(t ; q)]=0 .
\end{aligned}
$$

Now, we can write the Taylor series for $\tilde{S}(t ; q), \tilde{I}(t ; q)$ and $\tilde{R}(t ; q)$ with respect to $q$ in the following form

Table 3 Residual errors of $S_{5}(t), I_{5}(t)$ and $R_{5}(t)$ for different values of $\hbar$ 
Table 4 Residual errors of $S_{10}(t), I_{10}(t)$ and $R_{10}(t)$ for different values of $\hbar$

\begin{tabular}{|c|c|c|c|c|c|c|c|}
\hline$t$ & $\hbar=-1.2$ & $\hbar=-1.1$ & $\hbar_{\mathrm{opt}}=-1.0036$ & $\hbar=-1$ & $\hbar=-0.9$ & $\hbar=-0.8$ & $\hbar=-0.7$ \\
\hline 0.0 & $2.3552 \times 10^{-7}$ & $2.30233 \times 10^{-10}$ & $1.02141 \times 10^{-13}$ & $9.14824 \times 10^{-14}$ & $2.30053 \times 10^{-10}$ & $2.3552 \times 10^{-7}$ & 0.0000135813 \\
\hline 0.2 & $7.98087 \times 10^{-7}$ & $1.59804 \times 10^{-9}$ & $1.03917 \times 10^{-13}$ & $1.07914 \times 10^{-13}$ & $5.69842 \times 10^{-11}$ & $4.58415 \times 10^{-8}$ & $6.38727 \times 10^{-6}$ \\
\hline 0.4 & $1.85035 \times 10^{-6}$ & $5.17876 \times 10^{-9}$ & $9.81437 \times 10^{-14}$ & $1.23901 \times 10^{-13}$ & $5.71028 \times 10^{-11}$ & $4.56784 \times 10^{-8}$ & $1.45981 \times 10^{-6}$ \\
\hline 0.6 & $3.60403 \times 10^{-6}$ & $1.24299 \times 10^{-8}$ & $1.38556 \times 10^{-13}$ & $1.22125 \times 10^{-13}$ & $7.26663 \times 10^{-12}$ & $7.45872 \times 10^{-8}$ & $1.67784 \times 10^{-6}$ \\
\hline 0.8 & $6.30105 \times 10^{-6}$ & $2.47376 \times 10^{-8}$ & $5.59552 \times 10^{-14}$ & $1.66533 \times 10^{-13}$ & $1.12355 \times 10^{-12}$ & $6.8329 \times 10^{-8}$ & $3.44326 \times 10^{-6}$ \\
\hline 1.0 & 0.0000101995 & $4.26312 \times 10^{-8}$ & $1.3145 \times 10^{-13}$ & $3.46834 \times 10^{-13}$ & $3.20961 \times 10^{-11}$ & $4.69374 \times 10^{-8}$ & $4.19639 \times 10^{-6}$ \\
\hline$\|E\|$ & 0.0000101995 & $4.26312 \times 10^{-8}$ & $1.38556 \times 10^{-13}$ & $3.46834 \times 10^{-13}$ & $2.30053 \times 10^{-10}$ & $2.3552 \times 10^{-7}$ & 0.0000135813 \\
\hline$t$ & $\hbar=-1.2$ & $\hbar=-1.1$ & $\hbar_{\mathrm{opt}}=-1.0038$ & $\hbar=-1$ & $\hbar=-0.9$ & $\hbar=-0.8$ & $\hbar=-0.7$ \\
\hline 0.0 & $2.2528 \times 10^{-7}$ & $2.19797 \times 10^{-10}$ & $1.23457 \times 10^{-13}$ & $9.14824 \times 10^{-14}$ & $2.19932 \times 10^{-10}$ & $2.2528 \times 10^{-7}$ & 0.0000129908 \\
\hline 0.2 & $1.8875 \times 10^{-7}$ & $1.79984 \times 10^{-10}$ & $1.28342 \times 10^{-13}$ & $9.81437 \times 10^{-14}$ & $9.37903 \times 10^{-11}$ & $1.08779 \times 10^{-7}$ & $9.5656 \times 10^{-6}$ \\
\hline 0.4 & $3.0554 \times 10^{-7}$ & $2.84757 \times 10^{-9}$ & $1.32783 \times 10^{-13}$ & $1.01252 \times 10^{-13}$ & $3.81631 \times 10^{-10}$ & $5.43913 \times 10^{-8}$ & $4.78379 \times 10^{-6}$ \\
\hline 0.6 & $1.52798 \times 10^{-6}$ & $9.54972 \times 10^{-9}$ & $1.32561 \times 10^{-13}$ & $1.11244 \times 10^{-13}$ & $3.5494 \times 10^{-10}$ & $2.18038 \times 10^{-7}$ & $7.358 \times 10^{-7}$ \\
\hline 0.8 & $3.77689 \times 10^{-6}$ & $2.17536 \times 10^{-8}$ & $1.03695 \times 10^{-13}$ & $1.51879 \times 10^{-13}$ & $4.57945 \times 10^{-11}$ & $3.47132 \times 10^{-7}$ & $6.45201 \times 10^{-6}$ \\
\hline 1.0 & $7.35477 \times 10^{-6}$ & $3.95442 \times 10^{-8}$ & $3.39728 \times 10^{-14}$ & $4.14557 \times 10^{-13}$ & $7.11569 \times 10^{-10}$ & $4.17349 \times 10^{-7}$ & 0.0000119013 \\
\hline$\|E\|$ & $7.35477 \times 10^{-6}$ & $3.95442 \times 10^{-8}$ & $1.32783 \times 10^{-13}$ & $4.14557 \times 10^{-13}$ & $7.11569 \times 10^{-10}$ & $4.17349 \times 10^{-7}$ & 0.0000129908 \\
\hline$t$ & $\hbar=-1.3$ & $\hbar=-1.2$ & $\hbar=-1.1$ & $\hbar_{\mathrm{opt}}=-1$ & $\hbar=-0.9$ & $\hbar=-0.8$ & $\hbar=-0.7$ \\
\hline 0.0 & $2.95245 \times 10^{-6}$ & $5.11999 \times 10^{-8}$ & $5.00062 \times 10^{-11}$ & 0 & $5.00069 \times 10^{-11}$ & $5.12 \times 10^{-8}$ & $2.95245 \times 10^{-6}$ \\
\hline 0.2 & 0.0000203615 & $5.01628 \times 10^{-7}$ & $1.01934 \times 10^{-9}$ & $8.71525 \times 10^{-15}$ & $1.82436 \times 10^{-10}$ & $1.17196 \times 10^{-7}$ & $3.44612 \times 10^{-6}$ \\
\hline 0.4 & 0.0000446347 & $1.16719 \times 10^{-6}$ & $2.45188 \times 10^{-9}$ & $1.15463 \times 10^{-14}$ & $1.45817 \times 10^{-12}$ & $1.8454 \times 10^{-7}$ & $7.79626 \times 10^{-6}$ \\
\hline 0.6 & 0.0000746483 & $1.96985 \times 10^{-6}$ & $3.30358 \times 10^{-9}$ & $3.94129 \times 10^{-14}$ & $3.91076 \times 10^{-10}$ & $1.62374 \times 10^{-7}$ & 0.0000101862 \\
\hline 0.8 & 0.000108286 & $2.76035 \times 10^{-6}$ & $1.49223 \times 10^{-9}$ & $1.9984 \times 10^{-14}$ & $7.393 \times 10^{-10}$ & $6.72562 \times 10^{-8}$ & 0.0000107469 \\
\hline 1.0 & 0.000142277 & $3.30389 \times 10^{-6}$ & $6.33091 \times 10^{-9}$ & $1.97065 \times 10^{-14}$ & $8.26158 \times 10^{-10}$ & $8.0793 \times 10^{-8}$ & $9.64522 \times 10^{-6}$ \\
\hline$\|E\|$ & 0.000142277 & $3.30389 \times 10^{-6}$ & $6.33091 \times 10^{-9}$ & $3.94129 \times 10^{-14}$ & $8.26158 \times 10^{-10}$ & $1.8454 \times 10^{-7}$ & 0.0000107469 \\
\hline
\end{tabular}

$$
\begin{gathered}
\tilde{S}(t ; q)=S_{0}(t)+\sum_{m=1}^{\infty} S_{m}(t) q^{m}, \\
\tilde{I}(t ; q)=I_{0}(t)+\sum_{m=1}^{\infty} I_{m}(t) q^{m}, \\
\tilde{R}(t ; q)=R_{0}(t)+\sum_{m=1}^{\infty} R_{m}(t) q^{m},
\end{gathered}
$$

where

$$
\begin{gathered}
S_{m}=\left.\frac{1}{m !} \frac{\partial^{m} \tilde{S}(t ; q)}{\partial q^{m}}\right|_{q=0}, I_{m}=\left.\frac{1}{m !} \frac{\partial^{m} \tilde{I}(t ; q)}{\partial q^{m}}\right|_{q=0}, \\
R_{m}=\left.\frac{1}{m !} \frac{\partial^{m} \tilde{R}(t ; q)}{\partial q^{m}}\right|_{q=0} .
\end{gathered}
$$

For more analysis, the following vectors are defined

$$
\begin{aligned}
& \tilde{S}_{m}(t)=\left\{S_{0}(t), S_{1}(t), \ldots, S_{m}(t)\right\}, \\
& \tilde{I}_{m}(t)=\left\{I_{0}(t), I_{1}(t), \ldots, I_{m}(t)\right\}, \\
& \tilde{R}_{m}(t)=\left\{R_{0}(t), R_{1}(t), \ldots, R_{m}(t)\right\} .
\end{aligned}
$$

Differentiating Eq. (7) $m$-times with respect to $q$, dividing by $m$ ! and putting $q=0$ the $m$ th-order deformation equations can be obtained as follows

$$
\begin{gathered}
L_{S}\left[S_{m}(t)-\chi_{m} S_{m-1}(t)\right]=\hbar H_{S}(t) \Re_{m, S}\left(\mathbf{S}_{m-1}, \mathbf{I}_{m-1}, \mathbf{R}_{m-1}\right), \\
L_{I}\left[I_{m}(t)-\chi_{m} I_{m-1}(t)\right]=\hbar H_{I}(t) \Re_{m, I}\left(\mathbf{S}_{m-1}, \mathbf{I}_{m-1}, \mathbf{R}_{m-1}\right), \\
L_{R}\left[R_{m}(t)-\chi_{m} R_{m-1}(t)\right]=\hbar H_{R}(t) \Re_{m, R}\left(\mathbf{S}_{m-1}, \mathbf{I}_{m-1}, \mathbf{R}_{m-1}\right),
\end{gathered}
$$

where

$$
S_{m}(0)=0, I_{m}(0)=0, R_{m}(0)=0,
$$




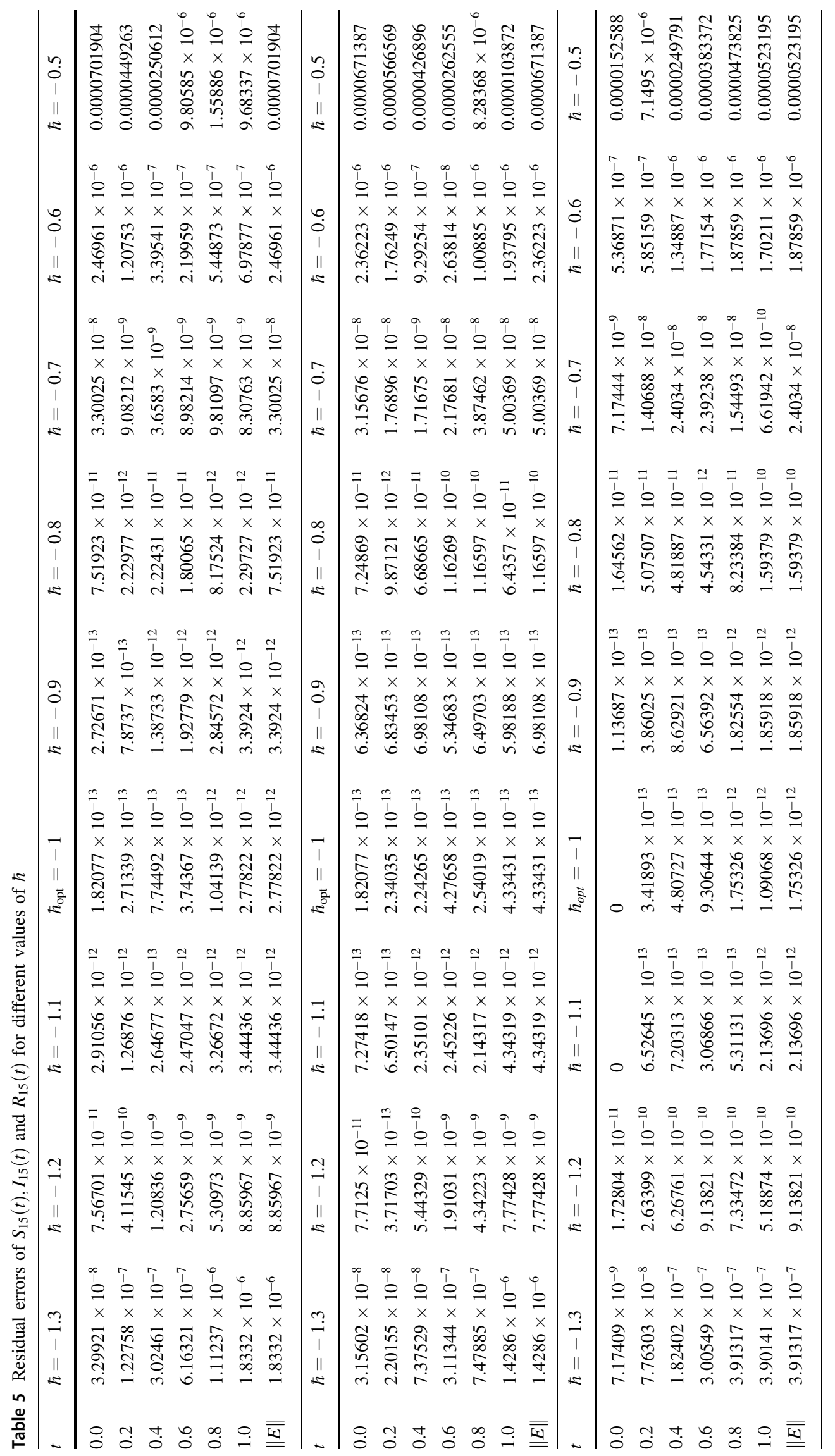




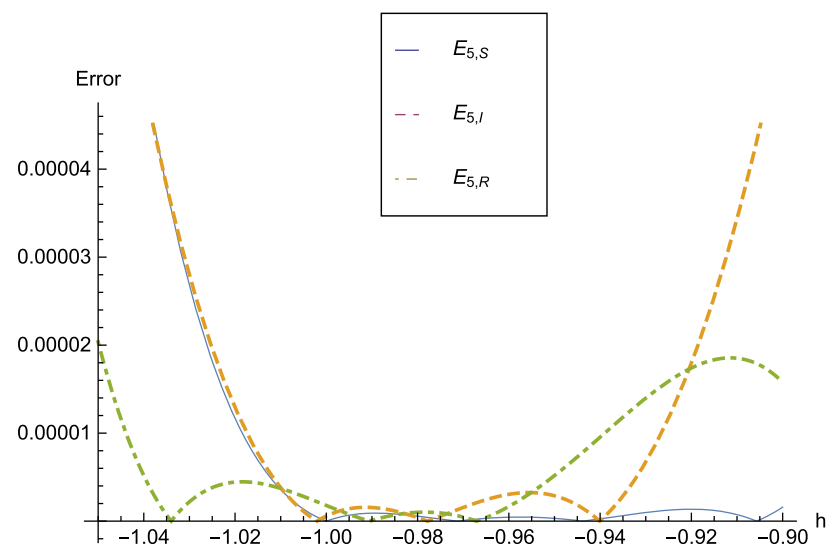

Fig. 4 Averaged residual errors $E_{5, S}, E_{5, I}, E_{5, R}$ versus $\hbar$ for $t=1$

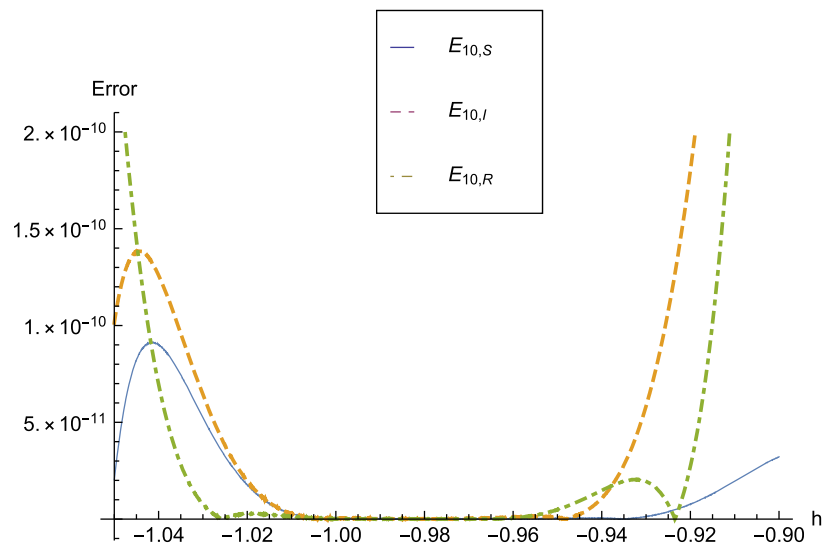

Fig. 5 Averaged residual errors $E_{10, S}, E_{10, I}, E_{10, R}$ versus $\hbar$ for $t=1$

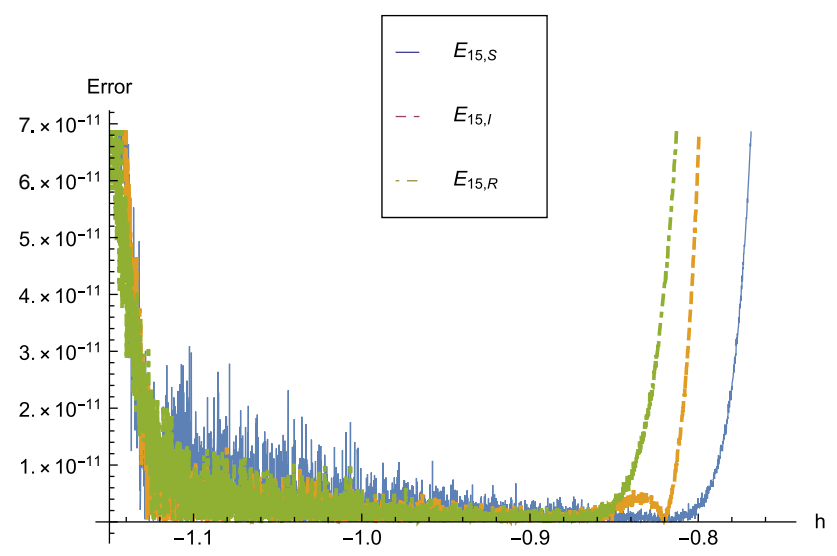

Fig. 6 Averaged residual errors $E_{15, S}, E_{15, I}, E_{15, R}$ versus $\hbar$ for $t=1$

$$
\begin{aligned}
& \Re_{m, S}(t)=\frac{\mathrm{d} S_{m-1}(t)}{\mathrm{d} t}-\left(1-\chi_{m}\right) f_{1}+\lambda \sum_{i=0}^{m-1} S_{i}(t) I_{m-1-i}(t) \\
& \quad+\mathrm{d} S_{m-1}(t), \\
& \Re_{m, I}(t)=\frac{\mathrm{d} I_{m-1}(t)}{\mathrm{d} t}-\left(1-\chi_{m}\right) f_{2}-\lambda \sum_{i=0}^{m-1} S_{i}(t) I_{m-1-i}(t) \\
& \quad+\varepsilon I_{m-1}(t)+\mathrm{d} R_{m-1}(t), \\
& \Re_{m, R}(t)=\frac{\mathrm{d} R_{m-1}(t)}{\mathrm{d} t}-\left(1-\chi_{m}\right) f_{3}-\varepsilon I_{m-1}(t)+\mathrm{d} R_{m-1}(t),
\end{aligned}
$$

and

$$
\chi_{m}= \begin{cases}0, & m \leq 1 \\ 1, & m>1 .\end{cases}
$$

By putting $H_{S}(t)=H_{I}(t)=H_{R}(t)=1$ and applying the inverse operators $L_{S}^{-1}, L_{I}^{-1}$ and $L_{R}^{-1}$, we have

$$
\begin{aligned}
S_{m}(t) & =\chi_{m} S_{m-1}(t)+\hbar \int_{0}^{t} \Re_{m, S}(t) \mathrm{d} t, \\
I_{m}(t) & =\chi_{m} I_{m-1}(t)+\hbar \int_{0}^{t} \Re_{m, I}(t) \mathrm{d} t, \\
R_{m}(t) & =\chi_{m} R_{m-1}(t)+\hbar \int_{0}^{t} \Re_{m, R}(t) \mathrm{d} t .
\end{aligned}
$$

Finally, the $m$ th-order approximate solution of nonlinear system (1) can be obtained as

$$
S_{m}(t)=\sum_{j=0}^{m} S_{j}(t), \quad I_{m}(t)=\sum_{j=0}^{m} I_{j}(t), \quad R_{m}(t)=\sum_{j=0}^{m} R_{j}(t) .
$$

\section{Numerical illustration}

In this section, the numerical results based on the HAM are presented. The approximate solutions for $m=5,10,15$ are obtained in the following form

and 


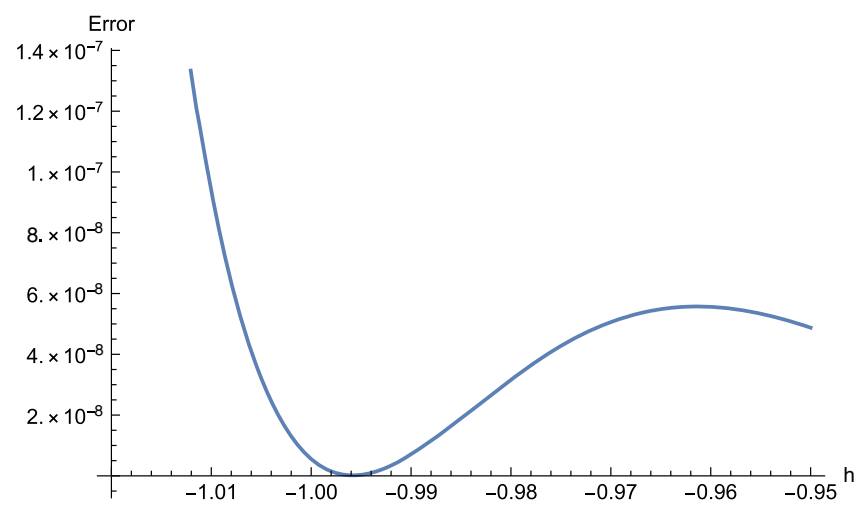

(A)

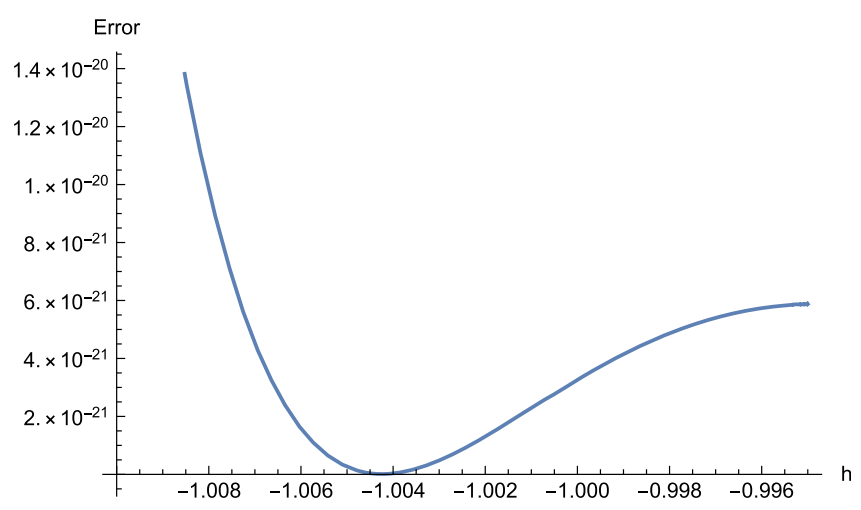

(B)

Fig. 7 Square residual errors of $\mathbf{a} E_{5, S}^{\prime}$ and $\mathbf{b} E_{10, S}^{\prime}$ versus $\hbar$ based on the OHAM

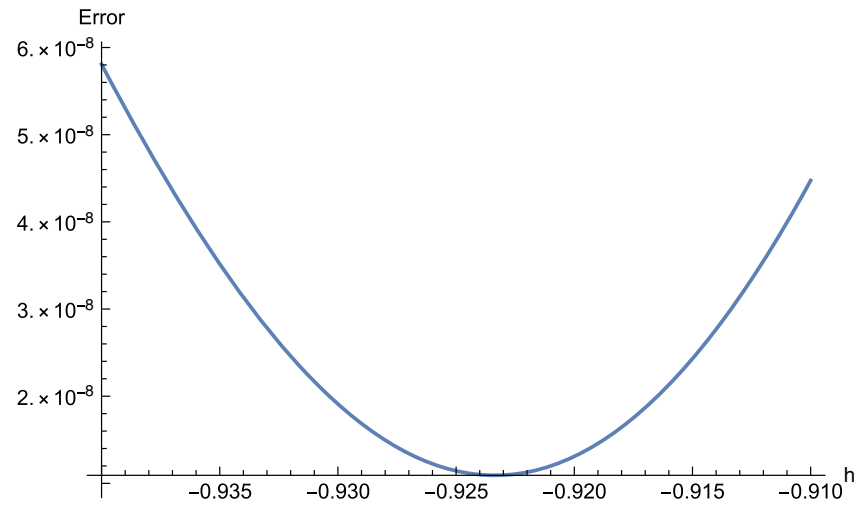

(A)

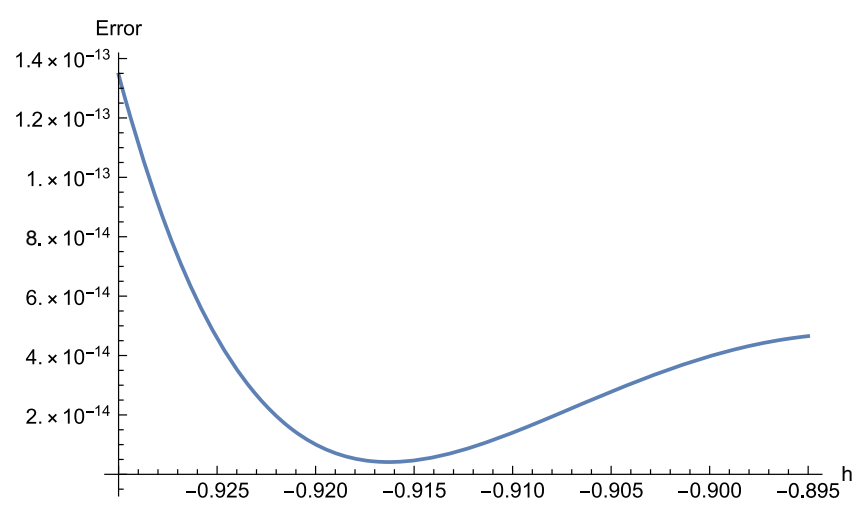

(B)

Fig. 8 Square residual errors of $\mathbf{a} E_{5, I}^{\prime}$ and $\mathbf{b} E_{10, I}^{\prime}$ versus $\hbar$ based on the OHAM

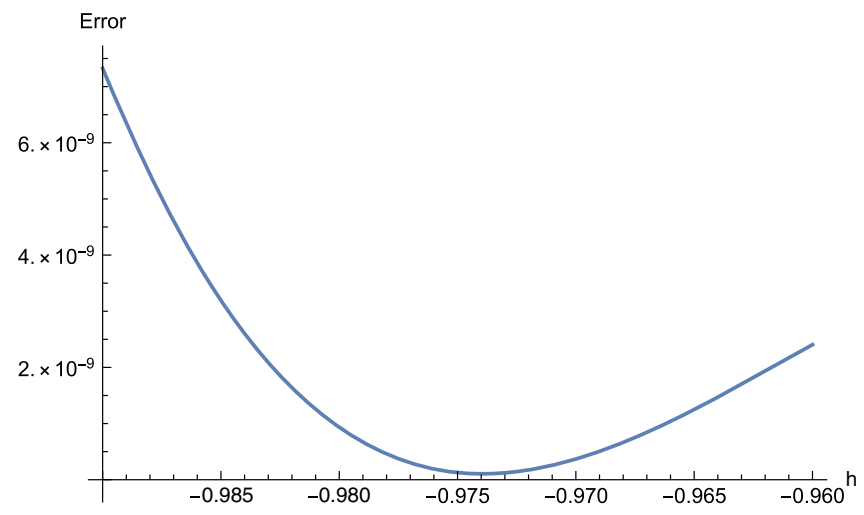

(A)

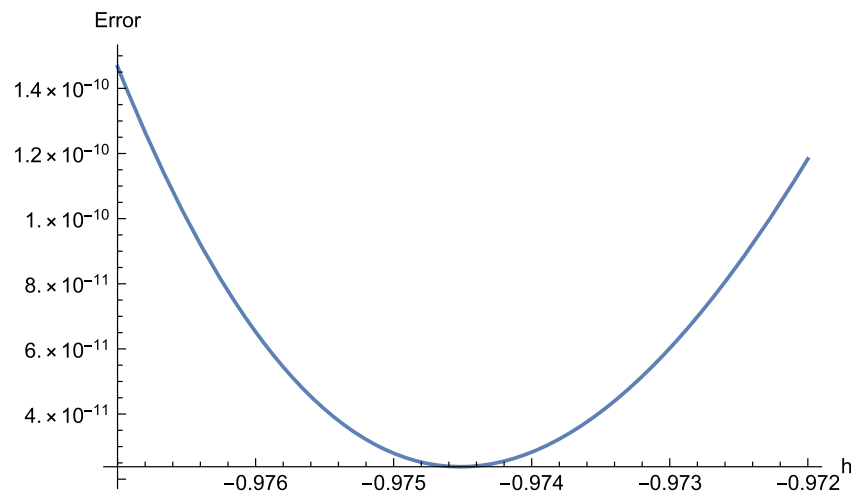

(B)

Fig. 9 Square residual errors of $\mathbf{a} E_{5, R}^{\prime}$ and $\mathbf{b} E_{10, R}^{\prime}$ versus $\hbar$ based on the OHAM 


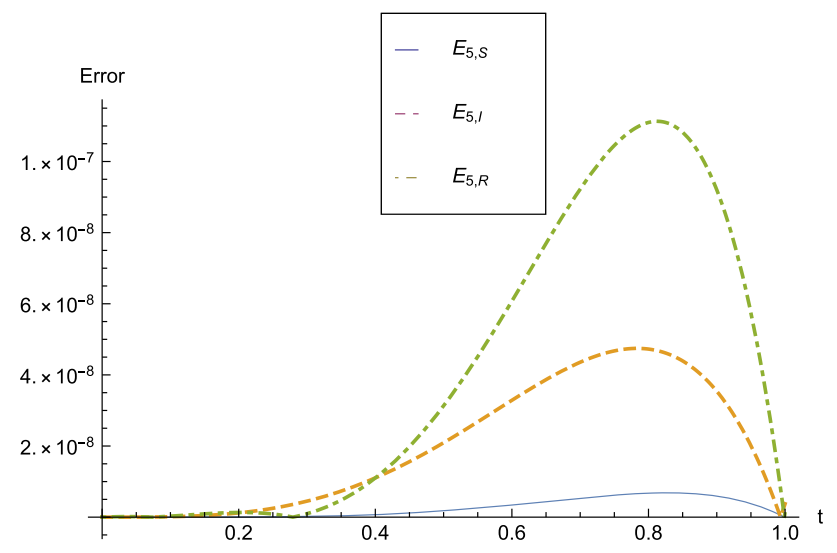

Fig. 10 Residual error functions for $E_{5, S}, E_{5, I}, E_{5, R}$ and the optimal values $\hbar^{*}$

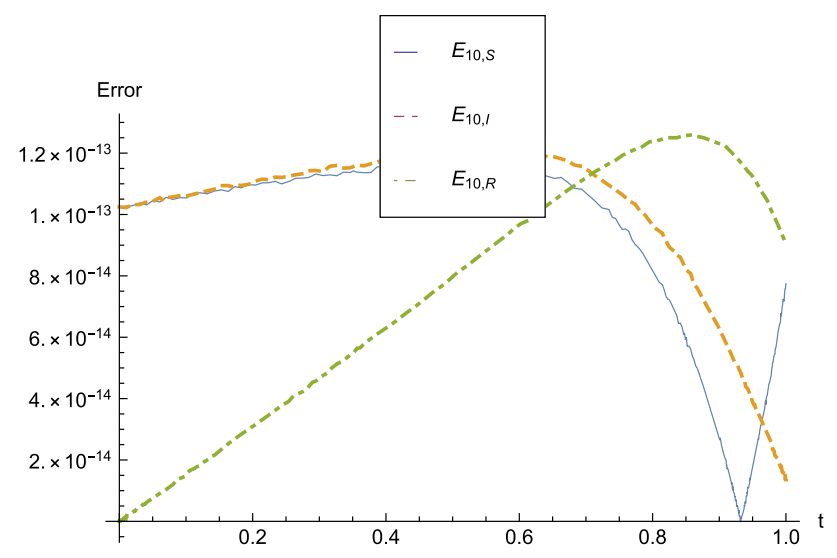

Fig. 11 Residual error functions for $E_{10, S}, E_{10, I}, E_{10, R}$ and the optimal values $\hbar^{*}$

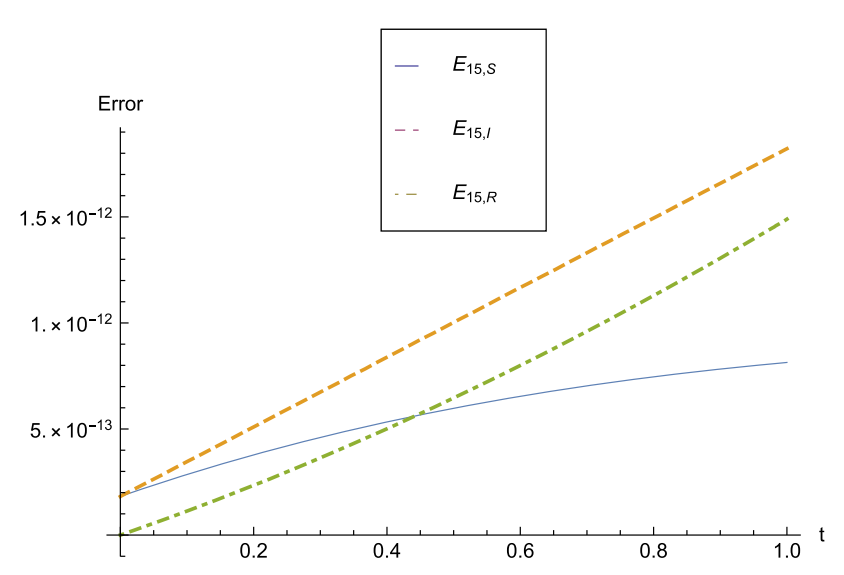

Fig. 12 Residual error functions for $E_{15, S}, E_{15, I}, E_{15, R}$ and the optimal values $\hbar^{*}$

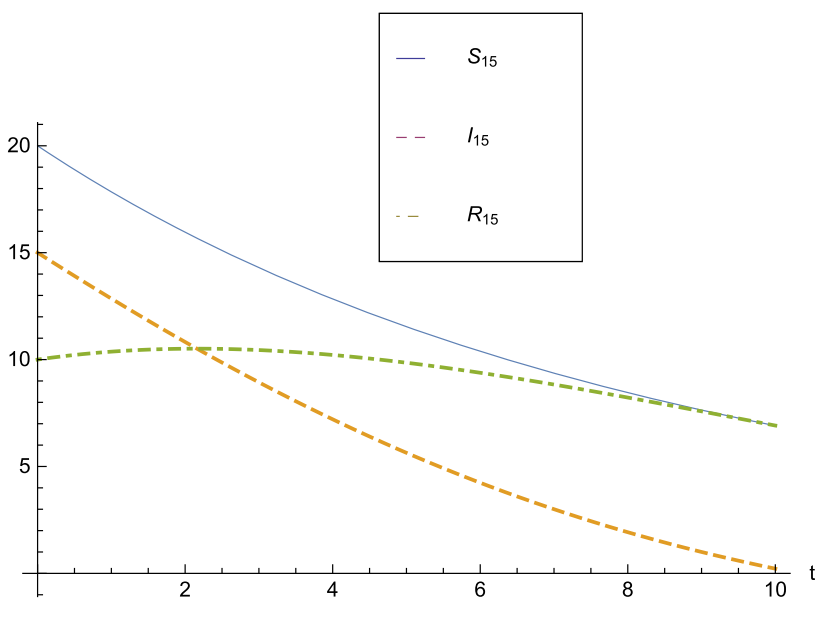

Fig. 13 Approximate solutions of $S(t), I(t)$ and $R(t)$ for $m=15, \hbar=$ -1 and $t \in[0,10]$

$$
\begin{aligned}
& S_{5}(t)=20+11.5 h t+23 h^{2} t+23 h^{3} t+11.5 h^{4} t+2.3 h^{5} t \\
& +1.5425 h^{2} t^{2}+3.085 h^{3} t^{2} \\
& +2.31375 h^{4} t^{2}+0.617 h^{5} t^{2}+0.0790458 h^{3} t^{3} \\
& +0.118569 h^{4} t^{3}+0.0474275 h^{5} t^{3} \\
& +0.00154855 h^{4} t^{4}+0.00123884 h^{5} t^{4} \\
& +7.74864 \times 10^{-6} h^{5} t^{5} \\
& I_{5}(t)=15+11 h t+22 h^{2} t+22 h^{3} t+11 h^{4} t+2.2 h^{5} t \\
& +0.4575 h^{2} t^{2}+0.915 h^{3} t^{2} \\
& +0.68625 h^{4} t^{2}+0.183 h^{5} t^{2}-0.0573792 h^{3} t^{3} \\
& -0.0860688 h^{4} t^{3}-0.0344275 h^{5} t^{3} \\
& -0.00203085 h^{4} t^{4}-0.00162468 h^{5} t^{4} \\
& -9.82135 \times 10^{-6} h^{5} t^{5} \\
& R_{5}(t)=10-2.5 h t-5 h^{2} t-5 h^{3} t-2.5 h^{4} t-0.5 h^{5} t \\
& -1.35 h^{2} t^{2}-2.7 h^{3} t^{2} \\
& -2.025 h^{4} t^{2}-0.54 h^{5} t^{2}-0.06025 h^{3} t^{3} \\
& -0.090375 h^{4} t^{3}-0.03615 h^{5} t^{3} \\
& -0.0000358854 h^{4} t^{4}-0.0000287083 h^{5} t^{4} \\
& +7.97984 \times 10^{-6} h^{5} t^{5}, \\
& S_{10}(t)=20+23 h t+103.5 h^{2} t+276 h^{3} t+483 h^{4} t \\
& +579.6 h^{5} t+483 h^{6} t+276 h^{7} t \\
& +\cdots-3.32927 \times 10^{-10} h^{9} t^{9}-2.99635 \times 10^{-10} h^{10} t^{9} \\
& -5.68453 \times 10^{-13} h^{10} t^{10}, \\
& I_{10}(t)=15+22 h t+99 h^{2} t+264 h^{3} t+462 h^{4} t \\
& +554.4 h^{5} t+462 h^{6} t+264 h^{7} t \\
& +\cdots+3.2067 \times 10^{-10} h^{9} t^{9}+2.88603 \\
& \times 10^{-10} h^{10} t^{9}+4.21668 \times 10^{-13} h^{10} t^{10},
\end{aligned}
$$



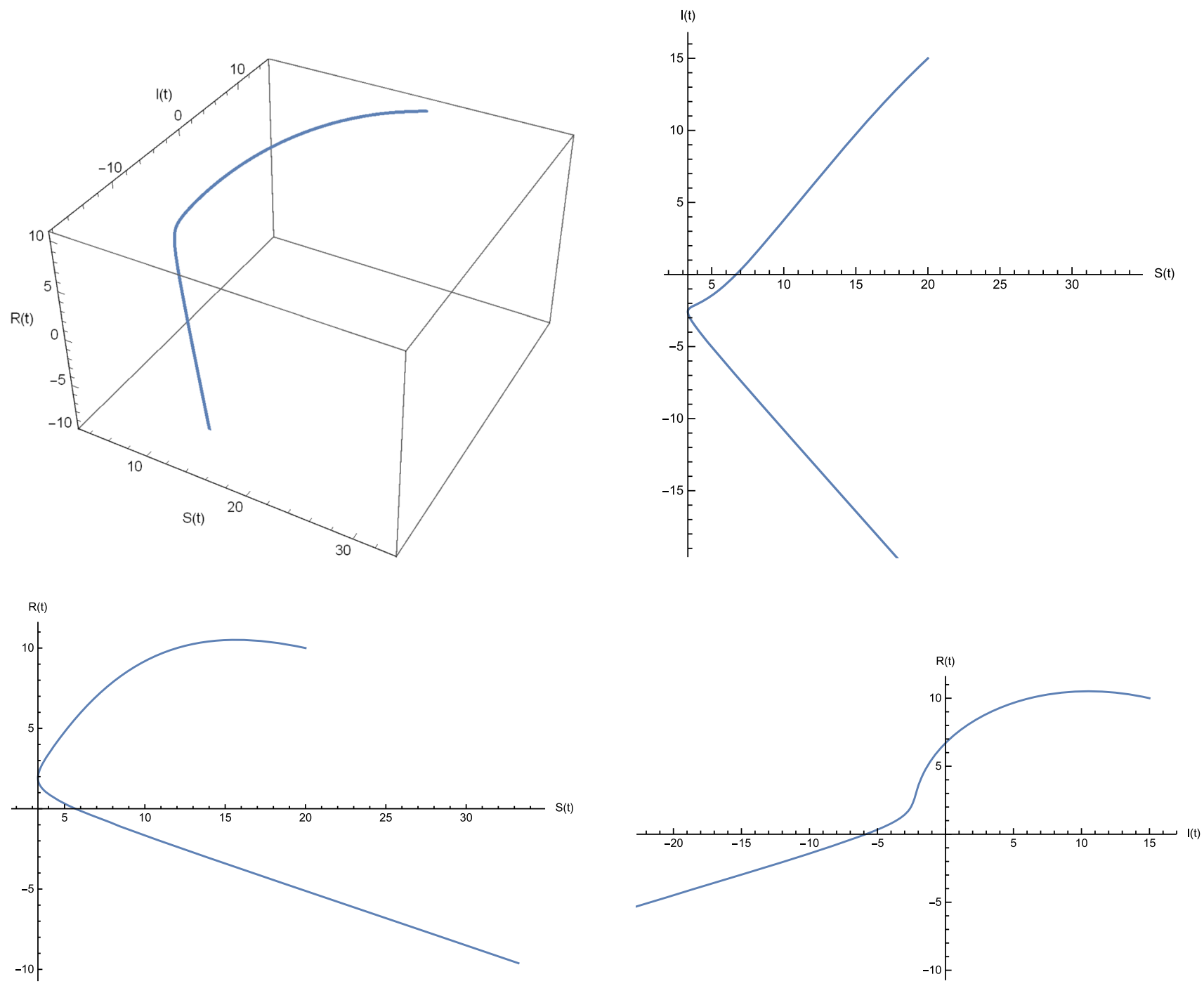

Fig. 14 Phase portraits of $S_{15}(t), I_{15}(t), R_{15}(t)$ by using the HAM

$$
\begin{aligned}
R_{10}(t)=10 & -5 h t-22.5 h^{2} t-60 h^{3} t-105 h^{4} t \\
& -126 h^{5} t-105 h^{6} t-60 h^{7} t \\
+ & \cdots-1.34528 \times 10^{-10} h^{9} t^{9}-1.21075 \times 10^{-10} h^{10} t^{9} \\
& -4.55198 \times 10^{-13} h^{10} t^{10}, \\
S_{15}(t)=20 & +34.5 h t+241.5 h^{2} t+1046.5 h^{3} t \\
& +3139.5 h^{4} t+6906.9 h^{5} t \\
+ & \cdots+5.68303 \times 10^{-17} h^{14} t^{14}+5.30416 \times 10^{-17} h^{15} t^{14} \\
& +1.56646 \times 10^{-20} h^{15} t^{15}, \\
I_{15}(t)=15+ & 33 h t+231 h^{2} t+1001 h^{3} t+3003 h^{4} t+6606.6 h^{5} t \\
+ & \cdots-5.44064 \times 10^{-17} h^{14} t^{14}-5.07793 \\
& \times 10^{-17} h^{15} t^{14}-7.423 \times 10^{-21} h^{15} t^{15}, \\
R_{15}(t)=10- & 7.5 h t-52.5 h^{2} t-227.5 h^{3} t-682.5 h^{4} t-1501.5 h^{5} t \\
+ & \cdots+1.61197 \times 10^{-17} h^{14} t^{14}+1.50451 \\
& \times 10^{-17} h^{15} t^{14}+3.13449 \times 10^{-20} h^{15} t^{15},
\end{aligned}
$$

where $\hbar$ is the convergence control parameter of the HAM. In order to show the regions of convergence, several $\hbar$ curves are demonstrated in Figs. 1, 2 and 3. These regions are the parallel parts of $\hbar$-curves with axiom $x$. The regions of convergence for $m=5,10,15$ and $t=1$ are presented in Table 2.

In order to show the efficiency and accuracy of presented method, the following residual error functions are applied as follows

$$
\begin{aligned}
& E_{m, S}(t)=S_{m}^{\prime}(t)-f_{1}+\lambda S_{m}(t) I_{m}(t)+\mathrm{d} S_{m}(t), \\
& E_{m, I}(t)=I_{m}^{\prime}(t)-f_{2}-\lambda S_{m}(t) I_{m}(t)+\varepsilon I_{m}(t)+\mathrm{d} R_{m}(t), \\
& E_{m, R}(t)=R_{m}^{\prime}(t)-f_{3}-\varepsilon I_{m}(t)+\mathrm{d} R_{m}(t),
\end{aligned}
$$

and the numerical results for different values of $t, m$ based on the presented convergence regions are obtained in 
Tables 3, 4 and 5. Also, the norm of residual errors $E_{m, S}(t), E_{m, I}(t)$ and $E_{m, R}(t)$ is given in these tables. In Figs. 4,5 and 6 the residual errors for $t=1$ and versus $\hbar$ are demonstrated. By using these figures, the optimal values of convergence control parameter $\hbar$ can be obtained which are presented in Table 2. Also, in Figs. 7, 8, 9, 10, 11 and 12 the plots of square residual errors

$$
\begin{aligned}
& E_{m, S}^{\prime}=\int_{0}^{+\infty}\left\{N_{S}\left[\sum_{j=0}^{m} S_{j}(\xi)\right]\right\}^{2} \mathrm{~d} \xi \\
& E_{m, I}^{\prime}=\int_{0}^{+\infty}\left\{N_{I}\left[\sum_{j=0}^{m} I_{j}(\xi)\right]\right\}^{2} \mathrm{~d} \xi \\
& E_{m, R}^{\prime}=\int_{0}^{+\infty}\left\{N_{R}\left[\sum_{j=0}^{m} R_{j}(\xi)\right]\right\}^{2} \mathrm{~d} \xi
\end{aligned}
$$

based on the optimal homotopy analysis method (OHAM) are shown. Figure 13 shows the approximate solution for $m=15$ and $t \in[0,10]$. In Fig. 14, the phase portraits of $S-I, S-R, I-R$ and $S-I-R$ for 15th-order approximation of the HAM and $\hbar=-1$ are shown.

\section{Conclusion}

In this study, the modified nonlinear SIR epidemiological model of computer viruses was illustrated and the HAM was applied to solve the presented model. It is important to note that in this method we have some auxiliary parameters and functions. One of these parameters is the convergence control parameter $\hbar$ which can be applied to adjust and control the convergence region of obtained solutions. Thus, by plotting several $\hbar$-curves and finding the regions of convergence, we showed the advantages and abilities of method. The residual errors were applied to show the efficiency and accuracy of method.

Open Access This article is distributed under the terms of the Creative Commons Attribution 4.0 International License (http://creative commons.org/licenses/by/4.0/), which permits unrestricted use, distribution, and reproduction in any medium, provided you give appropriate credit to the original author(s) and the source, provide a link to the Creative Commons license, and indicate if changes were made.

\section{References}

1. Abbasbandy, S.: Homotopy analysis method for heat radiation equations. Int. Commun. Heat Mass Transf. 34, 380-387 (2007)

2. Abbasbandy, S., Jalili, M.: Determination of optimal convergence-control parameter value in homotopy analysis method. Numer. Algor. 64(4), 593-605 (2013)
3. Abbasbandy, S., Shivanian, E.: Solution of singular linear vibrational BVPs by the homotopy analysis method. J. Numer. Math. Stoch. 1(1), 77-84 (2009)

4. Abbasbandy, S., Shivanian, E., Vajravelu, K.: Mathematical properties of $\$$ lhbar $\$$-curve in the frame work of the homotopy analysis method. Commun. Nonlinear Sci. Numer. Simulat. 16, 4268-4275 (2011)

5. Abbasbandy, S., Shivanian, E., Vajravelu, K., Kumar, S.: A new approximate analytical technique for dual solutions of nonlinear differential equations arising in mixed convection heat transfer in a porous medium. Int. J. Numer. Methods Heat Fluid Flow 27(2), 486-503 (2017)

6. Araghi, M.F., Behzadi, S.S.: Numerical solution of nonlinear Volterra-Fredholm integro-differential equations using Homotopy analysis method. J. Appl. Math. Comput. 37(1-2), 1-2 (2011)

7. Araghi, M.F., Fallahzadeh, A.: Explicit series solution of Boussinesq equation by homotopy analysis method. J. Am. Sci. 8(11), 448-452 (2012)

8. Ahmad Soltani, L., Shivanian, E., Ezzati, R.: Convection-radiation heat transfer in solar heat exchangers filled with a porous medium: exact and shooting homotopy analysis solution. Appl. Therm. Eng. 103, 537-542 (2016)

9. Cohen, F.: Computer viruses: theory and experiments. Comput. Secur. 6, 22-35 (1987)

10. Ellahi, R., Shivanian, E., Abbasbandy, S., Hayat, T.: Numerical study of magnetohydrodynamics generalized Couette flow of Eyring-Powell fluid with heat transfer and slip condition. Int. J. Numer. Methods Heat Fluid Flow 26(5), 1433-1445 (2016)

11. Ellahi, R., Shivanian, E., Abbasbandy, S., Rahman, S.U., Hayat, T.: Analysis of steady flows in viscous fluid with heat $/$ mass transfer and slip effects. Int. J. Heat Mass Transf. 55(23), 6384-6390 (2012)

12. Fariborzi Araghi, M.A., Fallahzadeh, A.: On the convergence of the homotopy analysis method for solving the Schrodinger equation. J. Basic Appl. Sci. Res. 2(6), 6076-6083 (2012)

13. Fariborzi Araghi, M.A., Noeiaghdam, S.: Fibonacci-regularization method for solving Cauchy integral equations of the first kind. Ain Shams Eng J. 8, 363-369 (2017)

14. Fariborzi Araghi, M., Noeiaghdam, S.: A novel technique based on the homotopy analysis method to solve the first kind Cauchy integral equations arising in the theory of airfoils. J. Interpolat. Approx. Sci. Comput. 1, 1-13 (2016)

15. Fariborzi Araghi, M.A., Noeiaghdam, S.: Homotopy analysis transform method for solving generalized Abel's fuzzy integral equations of the first kind. IEEE (2016). https://doi.org/10.1109/ CFIS.2015.7391645

16. Fariborzi Araghi, M.A., Noeiaghdam, S.: Homotopy regularization method to solve the singular Volterra integral equations of the first kind. Jordan J. Math. Stat. 11(1), 1-12 (2018)

17. Freihat, A.A., Zurigat, M., Handam, A.H.: The multi-step homotopy analysis method for modified epidemiological model for computer viruses. Afr. Mat. 26(3-4), 585-596 (2015)

18. Han, X., Tan, Q.: Dynamical behavior of computer virus on Internet. Appl. Math. Comput. 217, 2520-2526 (2010)

19. Kephart, J.O., Hogg, T., Huberman, B.A.: Dynamics of computational ecosystems. Phys. Rev. A 40(1), 404-421 (1998)

20. Kumar, D., Singh, J., Sushila, J.: Application of homotopy analysis transform method to fractional biological population model. Roman. Rep. Phys. 65, 63-75 (2013)

21. Kumar, S., Singh, J., Kumar, D., Kapoor, S.: New homotopy analysis transform algorithm to solve Volterra integral equation. Ain Shams Eng J. 5, 243-246 (2014)

22. Liao, S.J.: The proposed homotopy analysis techniques for the solution of nonlinear problems. Ph.D. Thesis, Shanghai Jiao Tong University, Shanghai (1992) (in English) 
23. Liao, S.J.: Beyond Perturbation: Introduction to Homotopy Analysis Method. CRC Press, Boca Raton (2003)

24. Liao, S.J.: On the homotopy analysis method for nonlinear problems. Appl. Math. Comput. 147, 499-513 (2004)

25. Liao, S.J.: Homotopy Analysis Method in Nonlinear Differential Equations. Higher Education Press, Beijing (2012)

26. Liao, S.J., Tan, Y.: A general approach to obtain series solutions of nonlinear differential equations. Stud. Appl. Math. 119, 297-355 (2007)

27. Mikaeilvand, N., Noeiaghdam, S.: Mean value theorem for integrals and its application on numerically solving of Fredholm integral equation of second kind with Toeplitz plus Hankel Kernel. Int. J. Ind. Math. 6, 351-360 (2014)

28. Noeiaghdam, S.: Numerical solution of \$N\$-th order Fredholm integro-differential equations by integral mean value theorem method. Int. J. Pure Appl. Math. 99(3), 277-287 (2015)

29. Noeiaghdam, S.: A novel technique to solve the modified epidemiological model of computer viruses. SeMA J. (2018). https:// doi.org/10.1007/s40324-018-0163-3

30. Noeiaghdam, S., Fariborzi Araghi, M.A., Abbasbandy, S.: Finding optimal convergence control parameter in the homotopy analysis method to solve integral equations based on the stochastic arithmetic. Numer Algorithm (2018). https://doi.org/ 10.1007/s11075-018-0546-7

31. Noeiaghdam, S., Zarei, E., Barzegar Kelishami, H.: Homotopy analysis transform method for solving Abel's integral equations of the first kind. Ain Shams Eng J. 7, 483-495 (2016)

32. Oztürk, Y., Gülsu, M.: Numerical solution of a modified epidemiological model for computer viruses. Appl. Math. Model. 39(23-24), 7600-7610 (2015)

33. Piqueira, J.R.C., de Vasconcelos, A.A., Gabriel, C.E.C.J., Araujo, V.O.: Dynamic models for computer viruses. Comput. Secur. 27, 355-359 (2008)

34. Ren, J., Yang, X., Yang, L., Xu, Y., Yang, F.: A delayed computer virus propagation model and its dynamics. Chaos Soliton Fractal 45, 74-79 (2012)
35. Ren, J., Yang, X., Zhu, Q., Yang, L., Zhang, C.: A novel computer virus model and its dynamics. Nonlinear Anal. Real. 3, 376-384 (2012)

36. Shaban, M., Shivanian, E., Abbasbandy, S.: Analyzing magnetohydrodynamic squeezing flow between two parallel disks with suction or injection by a new hybrid method based on the Tau method and the homotopy analysis method. Eur. Phys. J. Plus 128(11), 1-10 (2013)

37. Shivanian, E., Abbasbandy, S.: Predictor homotopy analysis method: two points second order boundary value problems. Nonlinear Anal. Real World Appl. 15, 89-99 (2014)

38. Shivanian, E., Alsulami, H.H., Alhuthali, M.S., Abbasbandy, S.: Predictor homotopy analysis method (pham) for nano boundary layer flows with nonlinear navier boundary condition: existence of four solutions. Filomat 28(8), 1687-1697 (2014)

39. Suleman, M., Lu, D., He, J.H., Farooq, U., Noeiaghdam, S., Chandio, F.A.: Elzaki projected differential transform method for fractional order system of linear and nonlinear fractional partial differential equation. Fractals (2018). https://doi.org/10.1142/ S0218348X1850041X

40. Wierman, J.C., Marchette, D.J.: Modeling computer virus prevalence with a susceptible-infected-susceptible model with reintroduction. Comput. Stat. Data Anal. 45, 3-23 (2004)

41. Zarei, E., Noeiaghdam, S.: Solving generalized Abel's integral equations of the first and second kinds via Taylor-collocation method. arXiv:1804.08571

42. Zhu, Q., Yang, X., Ren, J.: Modeling and analysis of the spread of computer virus. Commun. Nonlinear Sci. Numer. Simul. 17(12), 5117-5124 (2012)

Publisher's Note Springer Nature remains neutral with regard to jurisdictional claims in published maps and institutional affiliations. 